\title{
基于非线性输出频率响应函数的转子不对中-碰摩 耦合故障诊断方法研究
}

\author{
李志农 李云龙 刀海洋 \\ (南昌航空大学无损检测技术教育部重点实验室 南昌 330063)
}

\begin{abstract}
摘要 : 基于非线性输出频率响应函数(NOFRF)的独特优势, 将非线性输出频率响应函数引入到转子不对中-碰摩塊合故障中, 提 出了基于非线性输出频率响应函数的转子不对中-碰摩堣合故障沴断方法。利用提出的方法辨识得到了不同对中度及其角度的转 子系统的 NOFRF 值, 对比分析了不对中度及其角度对转子系统各阶 NOFRF 值的影响。仿真结果表明随着不对中度和角度的增 加, NOFRF 中二次谐波二阶非线性输出频率响应函数和二次谐波四阶非线性输出频率响应函数有着较为明显的增幅; 一次谐波 三阶非线性输出频率响应函数随不对中角度的增加而减小。因此，可以依据二次谐波二阶非线性输出频率响应函数、二次谐波 四阶非线性输出频率响应函数、一次谐波三阶非线性输出频率响应函数值的变化规律识别转子系统的不对中程度。最后通过试 验结果验证了仿真结果的正确性, 研究成果为具有不对中-碰摩堣合故障的转子系统的故障诊断提供了重要的依据。
\end{abstract}

关键词 : 非线性输出频率响应函数; 转子不对中 ; 碰摩; 故障诊断 ; 系统辨识

中图分类号 : TH17

\section{Research on Diagnosis Method of Rotor Misalignment-bumping Coupling Fault Based on Nonlinear Output Frequency Response Function}

\author{
LI Zhinong LI Yunlong DIAO Haiyang \\ (Key Laboratory of Nondestructive Testing, Ministry of Education, \\ Nanchang Hangkong University, Nanchang 330063)
}

\begin{abstract}
Based on the unique advantages of the nonlinear output frequency response function (NOFRF), the nonlinear output frequency response function is introduced to the rotor system with coupling faults of misalignment and rub-impact, a fault diagnosis method based on NOFRF for coupling faults of misalignment and rub-impact is proposed. The proposed method used to identify the NOFRF values of rotor systems with different degrees of misalignment and different angles, and the influence of misalignment and angle on the NOFRF values of various orders of the rotor system is analyzed. The simulation results show that the second harmonic second-order NOFRF value and the fourth-order NOFRF value have a significant increase with the increase of misalignment and angle. The first harmonic third-order NOFRF value decreases with the increase of misalignment angle. Therefore, the degree of misalignment of the rotor system can be identified based on the variation of the second harmonic second-order NOFRF value, the second harmonic fourth-order NOFRF value and the first harmonic third-order NOFRF value. Finally, the experimental results verify the correctness of the simulation results. The research results obtained provide an important evidence for fault diagnosis of rotor systems with coupling faults of misalignment and rub-impact.
\end{abstract}

Key words : nonlinear output frequency response function ; rotor misalignment ; rubbing ; fault diagnosis ; system identification

\section{0 前言}

耦合故障诊断在转子系统中是一难点, 相对于

\footnotetext{
* 国家自然科学基金 $(51675258,51075372,51265039)$ 、江西省教育厅科学 技术研究项目 (GJJ150699)和机械传动国家重点实验室开放基金 (SKLMT-KFKT-201514)资助项目。20180918 收到初稿 , 20190426 收到 修改稿
}

单一故障，复杂得多，也困难得多。现有的耦合故 障诊断主要停留在一些信号处理方法上 , 例如局融 合信息摘 ${ }^{[1]}$ 、经验模态分解 ${ }^{[2]}$ 、希尔伯特-黄变换 ${ }^{[3]}$ 、 灰色关联分析 ${ }^{[4]}$ 、分层模态综合法 ${ }^{[5]}$ 等。然而，这些 耦合故障诊断方法只是简单利用了系统的输出信 号，并无法表达整个系统的特征，因此在使用范围 上受到了限制。只有当系统的输入信号能够稳定保 持较长时间时, 这种方法才能取得令人满意的效果。 
其实, 系统输出的变化有可能是由于输入信号变化 所引起的, 而系统的传递函数和本质特征并没有发 生改变。在这种情况下, 如果只采用输出的变化来 判断系统是否发生了故障，就难免会发生错误。

基于此，人们提出了基于非线性模型的故障诊 断方法, 与基于信号分析的诊断方法的不同之处在 于，该方法在于不仅考虑了系统输出，而且也考虑 了系统的输入。其思路是根据检测系统的输入输出, 利用某种方法预测它们之间的映射关系, 即系统的 传递特性，根据系统的传递特性是否发生变化来判 断系统是否发生故障。非线性输出频率响应函数 (NOFRF) 是一种有效的非线性模型检测方法, 不仅 能反映系统输出频谱与非线性参数之间的关系, 而 且各阶次的函数都是一维的, 具有明确的物理意义, 也容易理解和接受。文献[6-12]已将 NOFRF 应用到 机械系统中，并取得了一些成果。然而，现有的 NOFRF 只是用于诊断单一故障, 并没有考虑多故障 耦合的 NOFRF 特性, 相对单一故障, 耦合故障的 研究更有意义, 不同故障之间相互影响，非线性问 题更加突出。基于此, 本文将 NOFRF 引入到耦合 故障诊断中, 以转子不对中-碰摩耦合故障为例, 通 过数值仿真, 研究分析不对中度及其角度对不对中碰摩耦合系统各阶 NOFRF 值的影响, 得到一些有 价值的结论，并通过试验结果进一步验证。

\section{NOFRF 辨识算法}

与线性系统相比, 非线性系统的输入与输出的 关系要更加复杂多变, 假定 $u(t), y(t)$ 分别表示系 统的输入和输出, 用 Volterra 级数可以将系统输入 与输出函数的关系描述为 ${ }^{[13]}$

$$
y(t)=\sum_{n=1}^{N} \int_{-\infty}^{+\infty} \cdots \int_{-\infty}^{+\infty} h_{n}\left(\tau_{1}, \cdots, \tau_{n}\right) \prod_{i=1}^{n} u\left(t-\tau_{i}\right) \mathrm{d} \tau_{i}
$$

式中, $h_{n}\left(\tau_{1}, \cdots, \tau_{n}\right)$ 代表第 $n$ 阶 Volterra 级数的核函 数， $N$ 为该系统的最高阶次， $\tau$ 为时延。

系统输出函数 $y(t)$ 的傅里叶变换表达式为

$$
Y(\mathrm{j} \omega)=\sum_{n=1}^{N} Y_{n}(\mathrm{j} \omega) \quad \forall \omega
$$

式中, $Y_{n}(\mathrm{j} \omega)$ 表示系统的 $n$ 阶输出频率响应函数， 其可通过下式计算得到

$$
\begin{gathered}
Y_{n}(\mathrm{j} \omega)=\frac{1 / \sqrt{n}}{(\boldsymbol{Z} \mathrm{T})^{n-1}} \times \int_{\omega_{1}+\cdots+\omega_{n}-\omega} H_{n}\left(\mathrm{j} \omega_{1}, \cdots, \mathrm{j} \omega_{n}\right) \times \\
\prod_{i=1}^{n} U\left(\mathrm{j} \omega_{i}\right) \mathrm{d} \sigma_{n \omega}
\end{gathered}
$$

式中, $U(\mathrm{j} \omega)$ 为系统输入函数 $u(t)$ 的傅里叶变换 ;
$H_{n}(\mathrm{j} \omega)$ 为该非线性系统 $n$ 阶非线性频率响应函数， 它可以表示为

$$
\begin{gathered}
H_{n}\left(\mathrm{j} \omega_{1}, \cdots, \mathrm{j} \omega_{n}\right)=\int_{-\infty}^{\infty} \cdots \int_{-\infty}^{\infty} h_{n}\left(\tau_{1}, \tau_{2}, \cdots, \tau_{n}\right) \times \\
\prod_{i=1}^{n} e^{-\mathrm{j} \omega_{i \sigma_{i}}} \mathrm{~d} \tau_{i}
\end{gathered}
$$

基于式(4)给出的广义频率响应函数 , 同时假设 $U_{n}(\mathrm{j} \omega)=\int_{\omega_{1}+\omega_{2}+\cdots+\omega_{n}=\omega} \prod_{i=1}^{n} U\left(\mathrm{j} \omega_{i}\right) \mathrm{d} \sigma_{n \omega} \neq 0$ ，得到可以 利用响应频率描述系统非线性特性的方法，得到非 线性输出频率响应函数

$$
G_{n}(\mathrm{j} \omega)=\frac{\int_{\omega_{1}+\cdots+\omega_{n}-\omega} H_{n}\left(\mathrm{j} \omega_{1}, \cdots, \mathrm{j} \omega_{n}\right) \prod_{i=1}^{n} U\left(\mathrm{j} \omega_{i}\right) \mathrm{d} \sigma_{n \omega}}{\int_{\omega_{1}+\cdots+\omega_{n}-\omega} \prod_{i=1}^{n} U\left(\mathrm{j} \omega_{i}\right) \mathrm{d} \sigma_{n \omega}}
$$

根据给定的非线性输出频率响应函数的定义, 将系统输出函数的傅里叶表达式改写为

$$
Y(\mathrm{j} \omega)=\sum_{n=1}^{N} Y_{n}(\mathrm{j} \omega)=\sum_{n=1}^{N} G_{n}(\mathrm{j} \omega) U_{n}(\mathrm{j} \omega)
$$

将式(6)写成矩阵形式

$$
[Y(\mathrm{j} \omega)]=\left[U_{1}(\mathrm{j} \omega), \cdots, U_{N}(\mathrm{j} \omega)\right][G(\mathrm{j} \omega)]
$$

式中 , $G(\mathrm{j} \omega)=\left[G_{1}(\mathrm{j} \omega), \cdots, G_{n}(\mathrm{j} \omega)\right]^{\mathrm{T}}$ 。

假设 $u(t)=\alpha u^{*}(t)$ ，且 $\alpha$ 是不为零的常数时， $u^{*}(t)$ 就表示系统的特定输入信号，即

$$
\begin{gathered}
U_{n}(\mathrm{j} \omega)=\frac{1 / \sqrt{n}}{(2 \mathrm{~T})^{n-1}} \int_{\omega_{1}+\cdots+\omega_{n}=\omega} \prod_{i=1}^{n} U\left(\mathrm{j} \omega_{j}\right) \mathrm{d} \sigma_{n \omega}= \\
\alpha^{n} \frac{1 / \sqrt{n}}{(2 \mathrm{~T})^{n-1}} \int_{\omega_{1}+\cdots+\omega_{n}=\omega} \prod_{i=1}^{n} U^{*}\left(\mathrm{j} \omega_{j}\right) \mathrm{d} \sigma_{n \omega}= \\
\alpha^{n} U_{n}^{*}(\mathrm{j} \omega)
\end{gathered}
$$

式中, $U^{*}(\mathrm{j} \omega)$ 是输入信号 $u^{*}(t)$ 的傅里叶变换, 且

$$
U_{n}^{*}(\mathrm{j} \omega)=\frac{1 / \sqrt{n}}{(2 \mathrm{~T})^{n-1}} \int_{\omega_{1}+\cdots+\omega_{n}=\omega} \prod_{i=1}^{n} U^{*}\left(\mathrm{j} \omega_{j}\right) \mathrm{d} \sigma_{n \omega}
$$

因此, 输入信号的傅里叶变换式可以改写为

$$
[Y(\mathrm{j} \omega)]=\left[\alpha U_{1}^{*}(\mathrm{j} \omega), \cdots, \alpha^{N} U_{N}^{*}(\mathrm{j} \omega)\right]^{*}
$$

$$
\left[G^{*}(\mathrm{j} \omega)\right]
$$

式中 , $\left[G^{*}(\mathrm{j} \omega)\right]=\left[G_{1}^{*}(\mathrm{j} \omega), \cdots, G_{N}^{*}(\mathrm{j} \omega)\right]^{\mathrm{T}}$, 表示为各阶 NOFRF 的估计矢量。

由式(10)可知，给定多于 $N$ 的 $M$ 次输入信号激 励，就可以求解式(10)中的 $G_{1}^{*}(\mathrm{j} \omega), \cdots, G_{N}^{*}(\mathrm{j} \omega)$ 。

一般, 转子系统受到的激励信号为谐波激励， 当转子系统的激励为 ${ }^{[14]}$

$$
u(t)=A \cos \left(\omega_{F} t+\beta\right)
$$

根据文献[18]所述，将输出函数的傅里叶变换 
重新改写为

$$
\begin{gathered}
Y(\mathrm{j} \omega)=\sum_{n=1}^{N} Y_{n}(\mathrm{j} \omega)= \\
\sum_{n=1}^{N}\left[2^{-n} \sum_{\omega_{k 1}+\ldots+\omega_{k n}=\omega} H_{n}\left(\mathrm{j} \omega_{k 1}, \cdots, \mathrm{j} \omega_{k n}\right) \times A\left(\mathrm{j} \omega_{k 1}\right) \cdots A\left(\mathrm{j} \omega_{k n}\right)\right]
\end{gathered}
$$

$$
A(\mathrm{j} \omega)=\left\{\begin{array}{l}
|A| \exp (\mathrm{jsign}(k) \beta) \omega \in\left\{k \omega_{F}, k= \pm 1\right\} \\
0 \quad \text { 其他 }
\end{array}\right.
$$

为了简化后面仿真与试验的计算过程, 在这里 假设系统的 $n$ 阶输出频率分量为 $\xi_{n}$, 即 ${ }^{[18]}$

$$
\xi=\bigcup_{n=1}^{N} \xi_{n}
$$

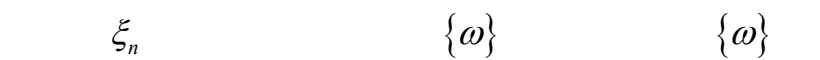

$$
\left\{\omega=\omega_{k 1}+\cdots+\omega_{k n} \mid \omega_{k i}=\omega_{F}, \quad i=1, \cdots, n\right\}
$$

将系统的 $n$ 阶输出频率分量进一步改写为

$$
\xi=\bigcup_{n=1}^{N} \xi_{n}=\left\{k \omega_{F}, k=-N, \cdots,-1,0,1, \cdots, N\right\}
$$

式中， $k$ 代表 $\omega_{k 1}, \cdots, \omega_{k n}$ 中等于 $\omega_{F}$ 的个数。

因此, 系统的输出频率 $Y(\mathrm{j} \omega)$ 和式(13)可以重写 为 ${ }^{[18]}$

$$
\begin{gathered}
Y(\mathrm{j} \omega)=\sum_{n=1}^{N} Y_{n}(\mathrm{j} \omega)=\sum_{n=1}^{N} G_{n}(\mathrm{j} \omega) A_{n}(\mathrm{j} \omega) \\
A_{n}(\mathrm{j} \omega)=A_{n}\left(\mathrm{j}(-n+2 k) \omega_{F}\right)= \\
\frac{1}{2} C_{n}^{k}|A|^{n} e^{\mathrm{j}(-n+2 k) \beta}
\end{gathered}
$$

随着系统阶次的变大, 辨识系统频率分量的计 算量成倍的增加。而且当倍频分量高于 4 倍频, 其 幅值特别小, 基本可以不考虑, 所以一般考虑的只 有前 4 阶的系统频率输出。根据前式, 可得到下列 公式

$$
\begin{gathered}
Y\left(\mathrm{j} \omega_{F}\right)=G_{1}\left(\mathrm{j} \omega_{F}\right) A_{1}\left(\mathrm{j} \omega_{F}\right)+G_{3}\left(\mathrm{j} \omega_{F}\right) A_{3}\left(\mathrm{j} \omega_{F}\right) \\
Y\left(\mathrm{j} 2 \omega_{F}\right)=G_{2}\left(\mathrm{j} 2 \omega_{F}\right) A_{2}\left(\mathrm{j} 2 \omega_{F}\right)+ \\
G_{4}\left(\mathrm{j} 2 \omega_{F}\right) A_{4}\left(\mathrm{j} 2 \omega_{F}\right) \\
Y\left(\mathrm{j} 3 \omega_{F}\right)=G_{3}\left(\mathrm{j} 3 \omega_{F}\right) A_{3}\left(\mathrm{j} 3 \omega_{F}\right) \\
Y\left(\mathrm{j} 4 \omega_{F}\right)=G_{4}\left(\mathrm{j} 4 \omega_{F}\right) A_{4}\left(\mathrm{j} 4 \omega_{F}\right)
\end{gathered}
$$

当使用两个频率相同的复杂信号激励系统时, 根据式(19) (22)的方法可以计算得到转子系统的 各阶 NOFRF 值。假定使用两个频率相同幅值不同 的正弦波激励信号 $\xi(1)$ 和 $\xi(2)$ 激励系统，系统的输 入频谱 $Y^{(1)}\left(\mathrm{j} \omega_{F}\right)$ 和 $Y^{(2)}\left(\mathrm{j} \omega_{F}\right)$ 可根据式(19)得到

$$
\left(\begin{array}{l}
Y^{(1)}\left(\mathrm{j} \omega_{F}\right) \\
Y^{(2)}\left(\mathrm{j} \omega_{F}\right)
\end{array}\right)=\left(\begin{array}{ll}
A_{1}^{(1)}\left(\mathrm{j} \omega_{F}\right) & A_{3}^{(1)}\left(\mathrm{j} \omega_{F}\right) \\
A_{1}^{(2)}\left(\mathrm{j} \omega_{F}\right) & A_{3}^{(2)}\left(\mathrm{j} \omega_{F}\right)
\end{array}\right)\left(\begin{array}{l}
G_{1}\left(\mathrm{j} \omega_{F}\right) \\
G_{3}\left(\mathrm{j} \omega_{F}\right)
\end{array}\right)
$$

类似的，其他各阶 NOFRF 值也可以由式(20) (22)可以得到。

\section{2 数值仿真}

在文献[19-21]的基础上，建立了具有不对中碰摩耦合故障的转子动力学模型, 该模型的示意图 如图 1 所示。

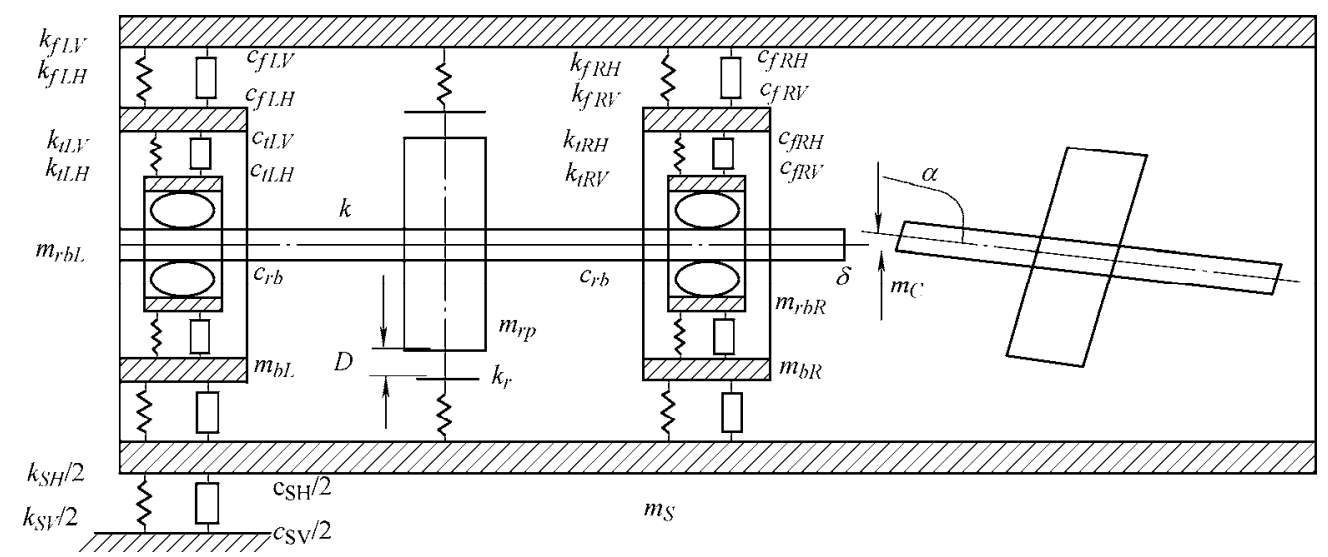

图 1 不对中-碰摩耦合故障的转子动力学模型

图 1 中， $m_{S}$ 为机匣(定子)的质量 ; $m_{r p} 、 m_{r b L}$ 、 $m_{r b R}$ 为转子系统质心位于圆盘和左、右两个轴承处 的等效质量 ; $m_{b L} 、 m_{b R}$ 是支撑左、右两个轴承支座 的质量; $m_{C}$ 为两个半联轴器的质量和; $k$ 为转子刚 度; $k_{r}$ 为靠近圆盘处的接触碰摩刚度; $k_{f L V} 、 k_{f L H}$ 为 机匣与左侧两个轴承支座间的垂向、横向支承刚度 ; $k_{f R V} 、 k_{f R H}$ 为机匣与右侧两个轴承支座间的垂向、 横向支承刚度 ; $k_{t L V} 、 k_{t L H}$ 为左侧两个轴承与支座间 的垂向、横向支承刚度 ; $k_{t R V} 、 k_{t R H}$ 为右侧两个轴 承与支座间的垂向、横向支承刚度 ; $c_{f L V} 、 c_{f L H}$ 为 机匣与左侧两个轴承支座间的垂向、横向阻尼系数; $c_{f R V} 、 c_{f R H}$ 为机匣与右侧两个轴承支座间的垂向、 
横向阻尼系数 $; c_{t L V} 、 c_{t L H}$ 为左侧两个轴承与支座间 的垂向、横向阻尼系数; $c_{t R V} 、 c_{t R H}$ 为右侧两个轴 承与支座间的垂向、横向阻尼系数; $k_{S V}$ 为机匣与基 座间的垂向支承刚度 ; $k_{S H}$ 为机匣与基座间的横向支 承刚度; $c_{S V}$ 为机匣与基座间的垂向阻尼系数; $c_{S H}$ 为 机匣与基座间的横向阻尼系数; $c_{r p} 、 c_{r b}$ 是圆盘和左、 右两个轴承处的阻尼系数; $\delta$ 为水平不对中量; $\alpha$ 为 角度不对中量 ; $D$ 为转子与定子之间的间隙。

依据牛顿第二定律, 建立的具有不对中-碰摩塊 合故障的转子动力学微分方程见式(24)

$$
\left\{\begin{array}{l}
m_{r p} \ddot{x}_{r p}+c_{r p} \dot{x}_{r p}+k\left(x_{r p}-x_{r b L}\right)+k\left(x_{r p}-x_{r b R}\right)=P_{x} \\
m_{r p} \ddot{y}_{r p}+c_{r p} \dot{y}_{r p}+k\left(y_{r p}-y_{r b L}\right)+k\left(y_{r p}-y_{r b R}\right)=P_{y}-m_{r p} g \\
m_{b R} \ddot{x}_{r b R}+c_{f R H}\left(\dot{x}_{r b R}-\dot{x}_{r p}\right)+c_{t R H}\left(\dot{x}_{r b R}-\dot{x}_{r p}\right)+ \\
\quad k_{f R H}\left(x_{r b R}-x_{r p}\right)+k_{t R H}\left(x_{r b R}-x_{r p}\right)=0 \\
m_{b R} \ddot{y}_{r b R}+c_{f R V}\left(\dot{y}_{r b R}-\dot{y}_{r p}\right)+c_{t R V}\left(\dot{y}_{r b R}-\dot{y}_{r p}\right)+ \\
\quad k_{f R V}\left(y_{r b R}-y_{r p}\right)+k_{t R V}\left(y_{r b R}-y_{r p}\right)=-m_{b R} g \\
m_{b L} \ddot{x}_{r b L}+c_{f L H}\left(\dot{x}_{r b L}-\dot{x}_{r p}\right)+c_{t L H}\left(\dot{x}_{r b L}-\dot{x}_{r p}\right)+ \\
\quad k_{f L H}\left(x_{r b L}-x_{r p}\right)+k_{t L H}\left(x_{r b L}-x_{r p}\right)=0 \\
m_{b L} \ddot{y}_{r b L}+c_{f L V}\left(\dot{y}_{r b L}-\dot{y}_{r p}\right)+c_{t L V}\left(\dot{y}_{r b L}-\dot{y}_{r p}\right)+ \\
\quad k_{f L V}\left(y_{r b L}-y_{r p}\right)+k_{t L V}\left(y_{r b L}-y_{r p}\right)=-m_{b L} g \\
m_{r b L} \ddot{x}_{r b L}+c_{r b} \dot{x}_{r b L}+k\left(x_{r b L}-x_{r p}\right)=F_{x L} \\
m_{r b L} \ddot{y}_{r b L}+c_{r b} \dot{y}_{r b L}+k\left(y_{r b L}-y_{r p}\right)=F_{y L}-m_{r b L} g \\
m_{r b L} \ddot{x}_{r b R}+c_{r b} \dot{x}_{r b R}+k\left(x_{r b R}-x_{r p}\right)=F_{x R} \\
m_{r b R} \ddot{y}_{r b R}+c_{r b} \dot{y}_{r b R}+k\left(y_{r b R}-y_{r p}\right)=F_{y R}-m_{r b R} g \\
m_{b L} \ddot{x}_{b L}+c_{f L H}\left(\dot{x}_{b L}-\dot{x}_{S}\right)+k_{f L H}\left(x_{b L}-x_{S}\right)=-F_{x L} \\
m_{b L} \ddot{y}_{b L}+c_{f L V}\left(\dot{y}_{b L}-\dot{y}_{S}\right)+k_{f L V}\left(y_{b L}-y_{S}\right)=-F_{y L}-m_{b L} g \\
m_{b R} \ddot{x}_{b R}+c_{f R H}\left(\dot{x}_{b R}-\dot{x}_{S}\right)+k_{f R H}\left(x_{b R}-x_{S}\right)=-F_{x R} \\
m_{b R} \ddot{y}_{b R}+c_{f R V}\left(\dot{y}_{b R}-\dot{y}_{S}\right)+k_{f R V}\left(y_{b R}-y_{S}\right)= \\
\quad-F_{y R}-m_{b R} g \\
m_{S} \ddot{x}_{S}+c_{S H} \dot{x}_{S}+k_{S H} x_{S}+c_{f L H}\left(\dot{x}_{S}-\dot{x}_{b L}\right)+ \\
\quad c_{f R H}\left(\dot{x}_{S}-\dot{x}_{b R}\right)+k_{f L H}\left(x_{S}-x_{b L}\right)+c_{f R H}\left(x_{S}-x_{b R}\right)= \\
\quad-P_{x} \\
m_{S} \ddot{y}_{S}+c_{S V} \dot{y}_{S}+k_{S V} y_{S}+c_{f L V}\left(\dot{y}_{S}-\dot{y}_{b L}\right)+ \\
\quad c_{f R V}\left(\dot{y}_{S}-\dot{y}_{b R}\right)+k_{f L V}\left(y_{S}-y_{b L}\right)+k_{f R V}\left(y_{S}-y_{b R}\right)= \\
\quad-P_{y}-m_{S} g \\
\end{array}\right.
$$

式中， $F_{x L}$ 和 $F_{y L}$ 为左轴承处的轴承力在 $x, y$ 方向的投 影, $F_{x R}$ 和 $F_{v R}$ 为右轴承处的轴承力在 $x, y$ 方向的投 影， $P_{x}$ 和 $P_{y}$ 为碰摩力在 $x, y$ 方向的投影。 $F_{x L}, F_{y L}$, $F_{x R} ， F_{y R} ， P_{x}$ 和 $P_{y}$ 的计算参考文献 [19-20]，即

$$
\left\{\begin{array}{l}
P_{x}=-k_{r}(1-D / r)\left(x_{r p}-\mu \cdot y_{r p}\right) \\
P_{y}=-k_{r}(1-D / r)\left(\mu \cdot x_{r p}+y_{r p}\right)
\end{array} \quad r \geqslant D\right.
$$

$$
\left\{\begin{array}{c}
F_{x}=\sum_{j=1}^{N_{b}} C_{b}\left(x \cos \theta_{j}+y \sin \theta_{j}-r_{0}\right)^{3 / 2} * \\
H\left(x \cos \theta_{j}+y \sin \theta_{j}-r_{0}\right) \cos \theta_{j} \\
F_{y}=\sum_{j=1}^{N_{b}} C_{b}\left(x \cos \theta_{j}+y \sin \theta_{j}-r_{0}\right)^{3 / 2} * \\
H\left(x \cos \theta_{j}+y \sin \theta_{j}-r_{0}\right) \sin \theta_{j}
\end{array}\right.
$$

式中, $\mu$ 为转静子之间的摩擦因数, $\mu=0.1 ; r$ 为 圆盘中心的径向位移, 即 : $r=\sqrt{x_{r p}^{2}+y_{r p}^{2}} ; C_{b}$ 为两 接触物体的赫兹接触刚度; $N_{b}$ 为滚珠个数 $; \theta_{j}$ 为 第 $j$ 个滚珠处的接触角度 $; r_{0}$ 为轴承间隙 $; H(t)$ 是 亥维赛函数。

这里, 选择参数 $\delta=0.2 \mathrm{~mm}, m_{r b L}=m_{r b R}=4.0 \mathrm{~kg}$, $m_{b L}=m_{b R}=20 \mathrm{~kg}, m_{r p}==32.1 \mathrm{~kg}, m_{S}=50 \mathrm{~kg}$, $k_{f L V}=k_{f L H}=k_{f R V}=k_{f R V}=7.5 \times 10^{7} \mathrm{~N} / \mathrm{m}, k=2.5 \times 10^{7}$ $\mathrm{N} / \mathrm{m} \quad, \quad k_{t L V}=k_{t L H}=k_{t R V}=k_{t R V}=7.5 \times 10^{7} \quad \mathrm{~N} / \mathrm{m}$, $k_{S V}=k_{S H}=3.5 \times 10^{9} \mathrm{~N} / \mathrm{m}, k_{r}=2.5 \times 10^{7} \mathrm{~N} / \mathrm{m}, D=0.02$ $\mathrm{mm}, c_{f L V}=c_{f L H}=c_{f R V}=c_{f R V}=2100 \mathrm{~N} \cdot \mathrm{s} / \mathrm{m}, c_{r p}=2100$ $\mathrm{N} \cdot \mathrm{s} / \mathrm{m}, c_{r b}=1050 \quad \mathrm{~N} \cdot \mathrm{s} / \mathrm{m}, \quad c_{t L V}=c_{t L H}=c_{t R V}=c_{t R V}=$ $1050 \mathrm{~N} \cdot \mathrm{s} / \mathrm{m}, c_{S V}=c_{S H}=2100 \mathrm{~N} \cdot \mathrm{s} / \mathrm{m}$ 。

在此, 先来分析不同不对中度和不同对中角度 情形下的频谱特性。图 2 为转速 $\omega=3500 \mathrm{r} / \mathrm{min}$ ，角 度 $\alpha=0.2 \mathrm{rad}$ 时 转子系统在不对中度 $\Delta E$ 分别为 0.1 $\mathrm{mm}, 0.2 \mathrm{~mm}, 0.3 \mathrm{~mm}$ 和 $0.5 \mathrm{~mm}$ 时的输出频谱图。

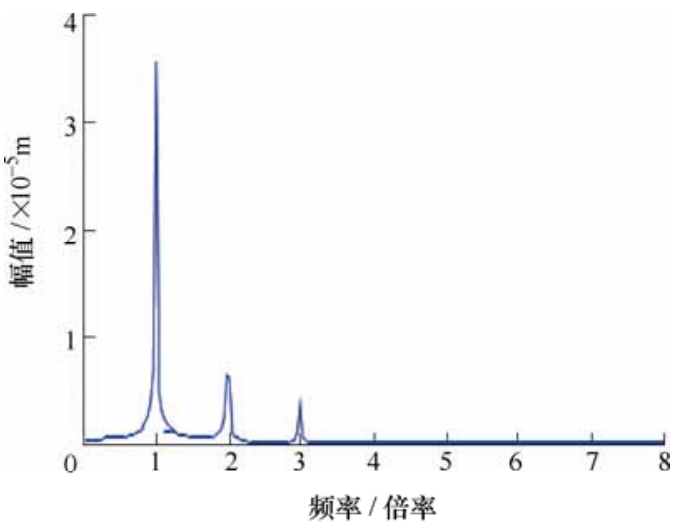

(a) $\Delta E=0.1 \mathrm{~mm}$

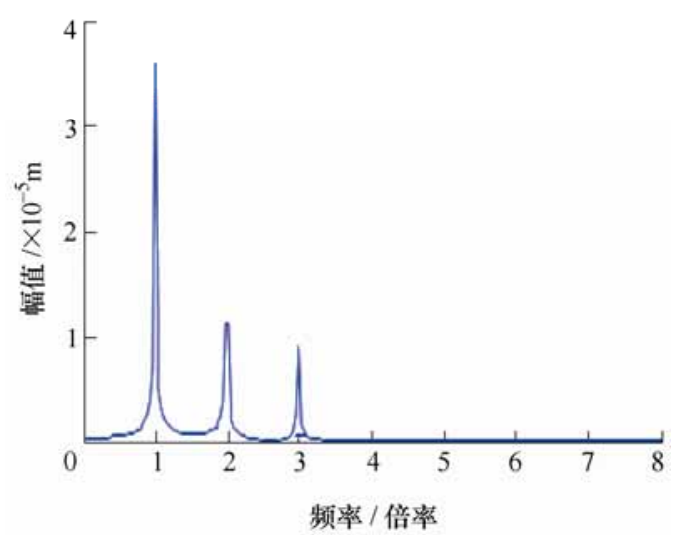

(b) $\Delta E=0.2 \mathrm{~mm}$ 


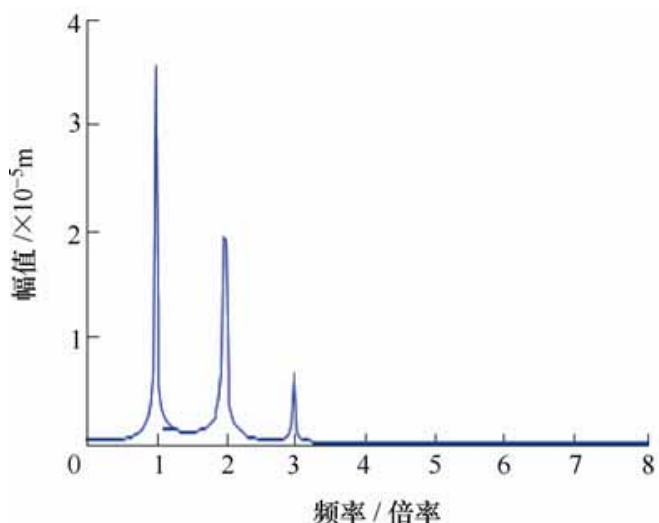

(c) $\Delta E=0.3 \mathrm{~mm}$

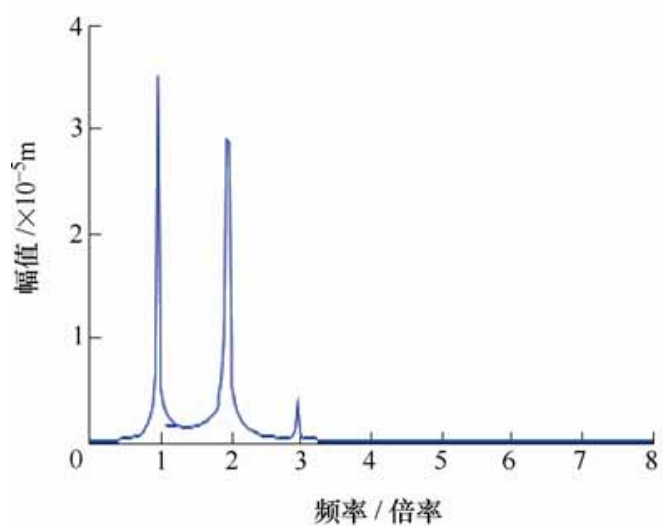

(d) $\Delta E=0.5 \mathrm{~mm}$

图 2 不同不对中度情形下系统输出频谱图

由图 2 可知，随着 $\Delta E$ 的变化，输出频谱特性 也在有规律地变化。当 $\Delta E=0.1 \mathrm{~mm}$ 时， 1 倍频分量 的幅值特别明显，但 2 倍频、3 倍频分量的幅值却 并不突出; 当 $\Delta E=0.2 \mathrm{~mm}$ 时, 1 倍频分量基本不变, 而 2 倍频、 3 倍频分量的幅值相较于 $\Delta E=0.1 \mathrm{~mm}$ 时 有了略微的增大 ; 当 $\Delta E$ 继续增大时, 2 倍频分量的 幅值增大更为显著, 但 3 倍频分量却稍微减小。总 之, 1 倍频分量的幅值基本不随着 $\Delta E$ 的变化而变 化, 2 倍频分量的幅值随 $\Delta E$ 的增加而增大，且增大 程度越来越大, 而 3 倍频分量的幅值随着 $\Delta E$ 的增 加先增大后变小

图 3 为转速 $\omega=3500 \mathrm{r} / \mathrm{min}, \Delta E=0.2 \mathrm{~mm}$ 时，不 同的不对中角度 $\alpha$ 的输出频谱图。与图 2 比较, 图 3 中开始有 4 倍频分量出现, 而且各倍频分量幅值 也比图 2 中的幅值大。当 $\alpha=15^{\circ}$ 时, 1 倍频分量相 比于其他多倍频分量尤为突出; 当 $\alpha=20^{\circ}$ 时, 与 $\alpha=15^{\circ}$ 时相比, 1 倍频分量的幅值略微减小, 2 倍频 分量的幅值有了明显的提升, 3 倍频和 4 倍频分量 虽然也有一定程度增加，但幅度并不太明显；当 $\alpha$ 分别为 $25^{\circ}$ 和 $30^{\circ}$ 时, 与 $\alpha$ 为 $15^{\circ}$ 和 $20^{\circ}$ 时结果相同, 1 倍频分量的幅值占比在逐渐减小, 2 倍频分量的幅

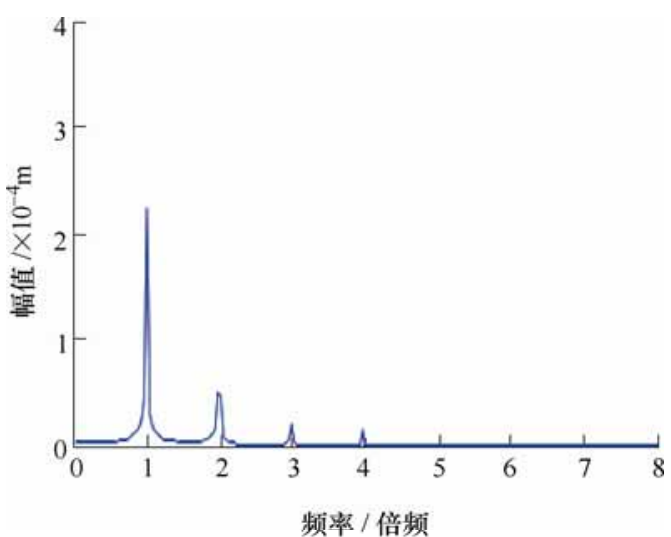

(a) $\alpha=15^{\circ}$

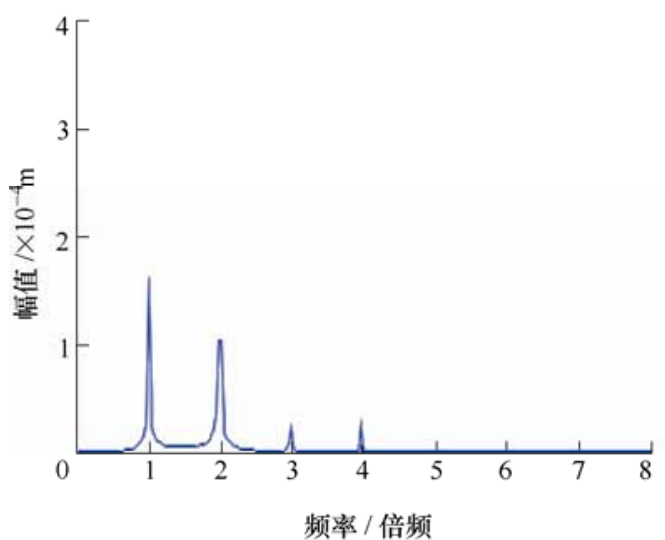

(b) $\alpha=20^{\circ}$

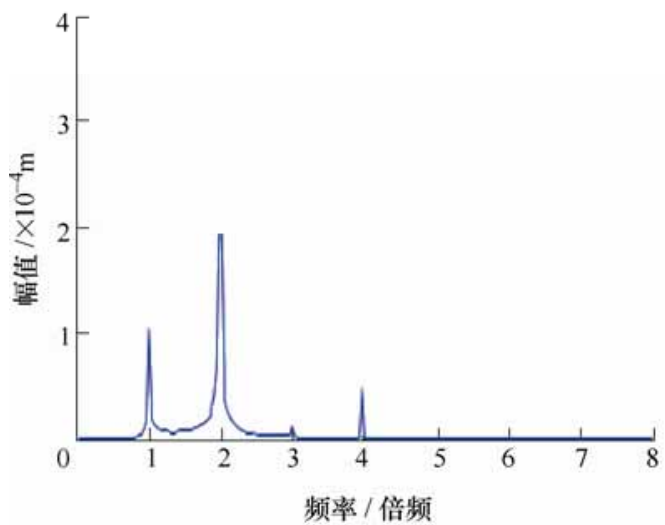

(c) $\alpha=25^{\circ}$

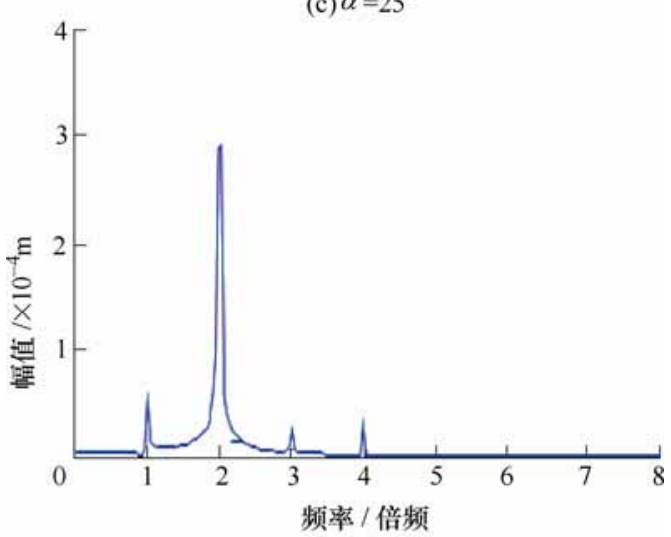

(d) $\alpha=30^{\circ}$

图 3 不同不对中角度情形下系统输出频谱图 
值增加非常显著，甚至超过了 1 倍频分量的幅值， 但是不同的是，3 倍频和 4 倍频分量有增有减，没 有规律可循。

再来分析不同不对中度和不同对中角度情形下 的 NOFRF 特性。表 1 为根据 NOFRF 理论计算得到 的不同不对中度下转子系统的各阶 NOFRF 值。观察 表 1 可以发现，随着不对中度 $\Delta E$ 从 $0.1 \mathrm{~mm}$ 增加到 $0.5 \mathrm{~mm}, \mathrm{NOFRF}$ 中的二次谐波二阶 NOFRF $\overline{G_{2}\left(\mathrm{j} 2 \omega_{F}\right)}$ 和二次谐波四阶 NOFRF $\overline{G_{4}\left(\mathrm{j} 2 \omega_{F}\right)}$ 也在增大，而且增 幅明显高于其他 NOFRF 值, 而系统的 2 阶响应主 要通过 $\overline{G_{2}\left(\mathrm{j} 2 \omega_{F}\right)}$ 和 $\overline{G_{4}\left(\mathrm{j} 2 \omega_{F}\right)}$ 两个成分反映, 因此 可以利用 $\overline{G_{2}\left(\mathrm{j} 2 \omega_{F}\right)}$ 和 $\overline{G_{4}\left(\mathrm{j} 2 \omega_{F}\right)}$ 的敏感性来判断转 子的不对中程度; 另外三次谐波三阶 NOFRF $\overline{G_{3}\left(\mathrm{j} 3 \omega_{F}\right)}$ 也随着不对中程度的增加也而增 大, 但是变化幅度比 $\overline{G_{2}\left(\mathrm{j} 2 \omega_{F}\right)}$ 和 $\overline{G_{4}\left(\mathrm{j} 2 \omega_{F}\right)}$ 小了很 多, 表明, 系统的三阶谐波也出现并重大, 表现在 它的三阶 NOFRF 上。其他各阶 NOFRF 值有增有减, 反映不是很明显。

表 1 不同不对中量情形下系统的 NOFRF 值

\begin{tabular}{cccccc}
\hline \multirow{2}{*}{ NOFRF } & \multirow{2}{*}{\begin{tabular}{c} 
无碰 \\
\cline { 3 - 6 }
\end{tabular}} & \multicolumn{5}{c}{ 不时对中度 $\Delta E / \mathrm{mm}$} \\
\hline$\overline{G_{1}\left(\mathrm{j} \omega_{F}\right)}$ & 1.0 & 1.0 & 1.0 & 1.0 & 1.0 \\
$\overline{G_{3}\left(\mathrm{j} \omega_{F}\right)}$ & 0 & $2.7 \times 10^{-5}$ & $8.2 \times 10^{-5}$ & $7.3 \times 10^{-5}$ & $1.8 \times 10^{-4}$ \\
$\overline{G_{2}\left(\mathrm{j} \omega_{F}\right)}$ & 0 & $8.3 \times 10^{-4}$ & $3.3 \times 10^{-3}$ & $9.3 \times 10^{-3}$ & $2.8 \times 10^{-2}$ \\
$\overline{G_{4}\left(\mathrm{j} 2 \omega_{F}\right)}$ & 0 & $7.2 \times 10^{-5}$ & $2.2 \times 10^{-4}$ & $4.1 \times 10^{-4}$ & $8.2 \times 10^{-3}$ \\
$\overline{G_{3}\left(\mathrm{j} 3 \omega_{F}\right)}$ & 0 & $6.6 \times 10^{-5}$ & $9.8 \times 10^{-5}$ & $2.6 \times 10^{-4}$ & $4.7 \times 10^{-4}$ \\
$\overline{G_{4}\left(\mathrm{j} 4 \omega_{F}\right)}$ & 0 & $6.4 \times 10^{-7}$ & $3.2 \times 10^{-6}$ & $8.3 \times 10^{-7}$ & $4.8 \times 10^{-6}$ \\
\hline
\end{tabular}

表 2 是在不同对中角度情形下系统的各阶 NOFRF 值。观察表 2 可以发现, 随着不对中角度的 不断增加, NOFRF 中二次谐波二阶 NOFRF $\overline{G_{2}\left(\mathrm{j} 2 \omega_{F}\right)}$ 、二次谐波四阶 NOFRF $\overline{G_{4}\left(\mathrm{j} 2 \omega_{F}\right)}$ 和四次 谐波四阶 NOFRF $\overline{G_{4}\left(\mathrm{j} 4 \omega_{F}\right)}$ 也在不断的大幅增大， 而 $\overline{G_{3}\left(\mathrm{j} \omega_{F}\right)}$ 则随之不断减小。转子系统的 4 阶响应 主要由 $\overline{G_{4}\left(\mathrm{j} 4 \omega_{F}\right)}$ 构成，因此，可以利用 $\overline{G_{4}\left(\mathrm{j} 4 \omega_{F}\right)}$ 的 变化来反映不对中角度的程度; 由于系统的 2 阶响 应主要通过 $\overline{G_{2}\left(\mathrm{j} 2 \omega_{F}\right)}$ 和 $\overline{G_{4}\left(\mathrm{j} 2 \omega_{F}\right)}$ 两个成分反映, 因此可以利用 $\overline{G_{2}\left(\mathrm{j} 2 \omega_{F}\right)}$ 和 $\overline{G_{4}\left(\mathrm{j} 2 \omega_{F}\right)}$ 的变化来反映 不对中角度的程度; 同样地, 系统的 1 阶响应主要 通过 $\overline{G_{1}\left(\mathrm{j} \omega_{F}\right)}$ 和 $\overline{G_{3}\left(\mathrm{j} \omega_{F}\right)}$ 两个成分反映, 但是由于 $\overline{G_{1}\left(\mathrm{j} \omega_{F}\right)}$ 保持不变, 只有 $\overline{G_{3}\left(\mathrm{j} \omega_{F}\right)}$ 随着不对中角度的 变化而变化, 因此可根据 $\overline{G_{3}\left(\mathrm{j} \omega_{F}\right)}$ 的变化来反映不 对中角度的程度。
表 2 不同不对中角度情形下系统的 NOFRF 值

\begin{tabular}{cccccc}
\hline \multirow{2}{*}{ NOFRF } & 无碰 & \multicolumn{4}{c}{ 角度 $/\left(^{\circ}\right)$} \\
\cline { 3 - 6 } & 摩时 & 15 & 20 & 25 & 30 \\
\hline$\overline{G_{1}\left(\mathrm{j} \omega_{F}\right)}$ & 1.0 & 1.0 & 1.0 & 1.0 & 1.0 \\
$\overline{G_{3}\left(\mathrm{j} \omega_{F}\right)}$ & 0 & $8.8 \times 10^{-3}$ & $1.2 \times 10^{-3}$ & $5.3 \times 10^{-4}$ & $8.6 \times 10^{-5}$ \\
$\overline{G_{2}\left(\mathrm{j} \omega_{F}\right)}$ & 0 & $8.3 \times 10^{-3}$ & $3.3 \times 10^{-2}$ & $8.4 \times 10^{-2}$ & $5.8 \times 10^{-1}$ \\
$\overline{G_{4}\left(\mathrm{j} 2 \omega_{F}\right)}$ & 0 & $6.2 \times 10^{-4}$ & $5.1 \times 10^{-3}$ & $1.1 \times 10^{-2}$ & $8.2 \times 10^{-2}$ \\
$\overline{G_{3}\left(\mathrm{j} 3 \omega_{F}\right)}$ & 0 & $6.6 \times 10^{-5}$ & $9.6 \times 10^{-5}$ & $8.5 \times 10^{-5}$ & $3.7 \times 10^{-5}$ \\
$\overline{G_{4}\left(\mathrm{j} 4 \omega_{F}\right)}$ & 0 & $5.4 \times 10^{-6}$ & $9.2 \times 10^{-6}$ & $1.3 \times 10^{-5}$ & $4.8 \times 10^{-5}$ \\
\hline
\end{tabular}

\section{3 试验研究}

为验证仿真结果的正确性, 在此, 在转子试验 台上设定了 3 种不对中情况进行不对中-碰摩耦合 故障试验, 分别称为轻微不对中、中度不对中和严 重不对中。

图 4 为试验得到的系统输出频谱图。由图 4 可 知, 当不对中比较轻微时, 图中的 1 倍频分量幅值 远高于其他倍频分量幅值，2 倍频分量的幅值偏小， 而 3 倍频和 4 倍频分量虽然也出现在图中，但是幅 值太小。当达到中度不对中时, 2 倍频分量幅值有 了较为明显的增大, 1 倍频分量所占的比例明显比

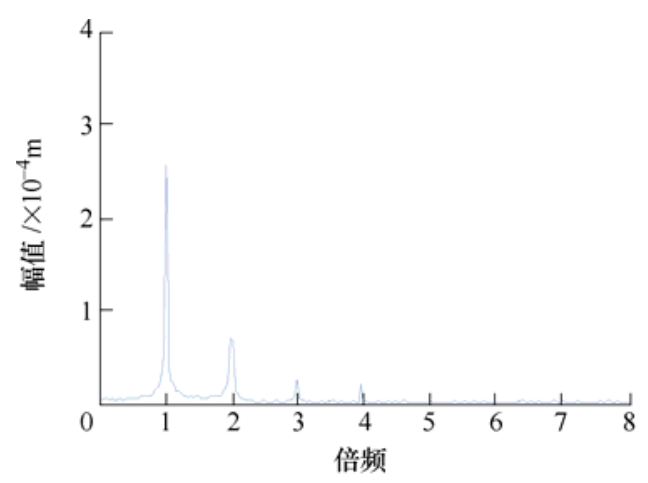

(a) 轻微不对中

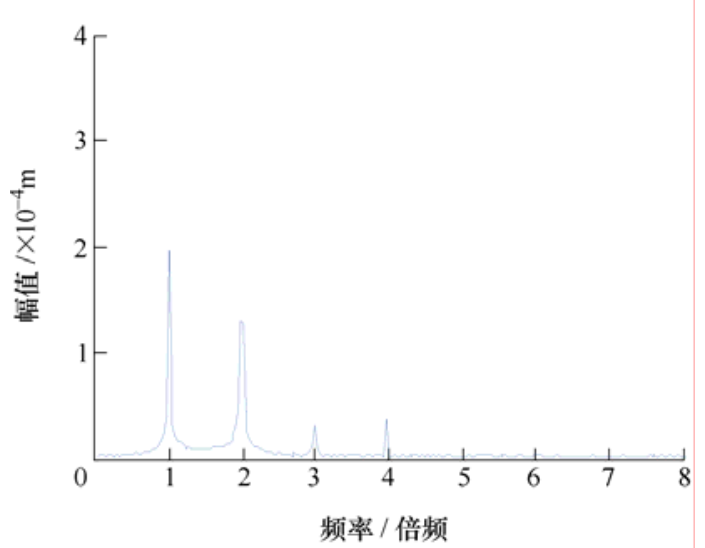

(b) 中度不对中 


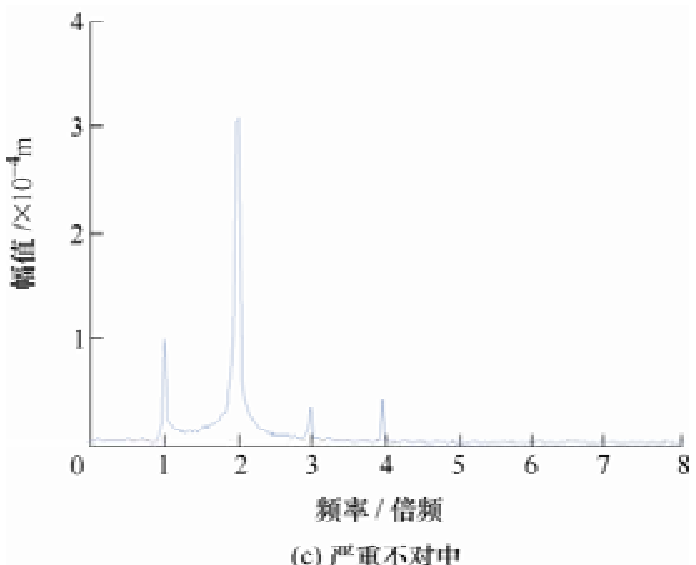

图 4 不同不对中度下系统输出频谱图

轻微不对中有一定程度的减小, 3 倍频和 4 倍频分 量相对 2 倍频仍比较弱; 当达到重度不对中时, 2 倍频分量幅值继续增大, 3 倍频和 4 倍频分量也明 显反映出。由此可见, 随着不对中的增大, 2 倍频 分量有明显的增大趋势; 3 倍频和 4 倍频分量也随 着不对中程度的变化也会随之出现, 并逐渐增大, 而，1 倍频分量所占比重会逐渐减少。

表 3 为试验得到的系统各阶 NOFRF 值,由表 3 可知, 随着不对中度程度的不断增加, NOFRF 中二 次谐波二阶 NOFRF $\overline{G_{2}\left(\mathrm{j} 2 \omega_{F}\right)}$ 、二次谐波四阶 NOFRF $\overline{G_{4}\left(\mathrm{j} 2 \omega_{F}\right)}$ 和 一次谐波三阶 NOFRF $\overline{G_{3}\left(\mathrm{j} \omega_{F}\right)}$ 都有相当明显的变化趋势 ; 其中 $\overline{G_{2}\left(\mathrm{j} 2 \omega_{F}\right)}$ 和 $\overline{G_{4}\left(\mathrm{j} 2 \omega_{F}\right)}$ 随着不对中程度的增加而增大，反映特 别明显。同样，验证了可以用 $\overline{G_{2}\left(\mathrm{j} 2 \omega_{F}\right)} 、 \overline{G_{4}\left(\mathrm{j} 2 \omega_{F}\right)}$ 来反映不对中的变化程度。

表 3 不同的不对中情形下系统的 NOFRF 值

\begin{tabular}{ccccc}
\hline NOFRF & 无碰摩时 & 轻微 & 中度 & 严重 \\
\hline$\overline{G_{1}\left(\mathrm{j} \omega_{F}\right)}$ & 1.0 & 1.0 & 1.0 & 1.0 \\
$\overline{G_{3}\left(\mathrm{j} \omega_{F}\right)}$ & 0 & $8.8 \times 10^{-3}$ & $2.2 \times 10^{-3}$ & $3.6 \times 10^{-4}$ \\
$\overline{G_{2}\left(\mathrm{j} \omega_{F}\right)}$ & 0 & $8.3 \times 10^{-3}$ & $3.3 \times 10^{-2}$ & $5.8 \times 10^{-1}$ \\
$\overline{G_{4}\left(\mathrm{j} 2 \omega_{F}\right)}$ & 0 & $6.2 \times 10^{-4}$ & $5.1 \times 10^{-3}$ & $8.2 \times 10^{-2}$ \\
$\overline{G_{3}\left(\mathrm{j} 3 \omega_{F}\right)}$ & 0 & $6.6 \times 10^{-6}$ & $9.6 \times 10^{-6}$ & $3.7 \times 10^{-6}$ \\
$\overline{G_{4}\left(\mathrm{j} 4 \omega_{F}\right)}$ & 0 & $3.4 \times 10^{-6}$ & $7.2 \times 10^{-6}$ & $8.8 \times 10^{-6}$ \\
\hline
\end{tabular}

\section{4 结论}

非线性输出频率响应函数是针对振动系统的非 线性模型检测的一种有效方法。本文将该方法引入 到耦合故障诊断中，提出了基于非线性输出频率响 应函数的多故障耦合诊断方法, 以对转子系统的不 对中和碰摩耦合故障为例, 建立了转子不对中-碰摩 耦合故障模型, 并在此基础上进行仿真和试验研究,
得到的结论如下。

(1) NOFRF 是一种有效的非线性振动检测方 法，该方法能够正确地反映系统的动态特性，对不 对中程度反映敏感，利用 NOFRF 对不对中的敏感 性，可以有效地诊断不对中的严重程度。

(2) NOFRF 对不对中量的变化反映明显, 随着 不对中量的增大, NOFRF 中的 $\overline{G_{2}\left(\mathrm{j} 2 \omega_{F}\right)}$ 和 $\overline{G_{4}\left(\mathrm{j} 2 \omega_{F}\right)}$ 反映明显，因此，可以利用 $\overline{G_{2}\left(\mathrm{j} 2 \omega_{F}\right)}$ 和 $\overline{G_{4}\left(\mathrm{j} 2 \omega_{F}\right)}$ 对不对中量的敏感性来定量判断平行不 对中的严重程度。

(3) NOFRF 对角度不对中也反映明显, 随着不 对中度角度的不断增大 , NOFRF 中的二次谐波二阶 NOFRF $\overline{G_{2}\left(\mathrm{j} 2 \omega_{F}\right)}$ 和二次谐波四阶 $\operatorname{NOFRF} \overline{G_{4}\left(\mathrm{j} 2 \omega_{F}\right)}$ 增大非常显著，同时随着不对中角 度的不断增加，NOFRF 中的一次谐波三阶 NOFRF $\overline{G_{3}\left(\mathrm{j} \omega_{F}\right)}$ 会不同程度地减小。因而可以通过 $\overline{G_{2}\left(\mathrm{j} 2 \omega_{F}\right)} 、 \overline{G_{4}\left(\mathrm{j} 2 \omega_{F}\right)}$ 和 $\overline{G_{3}\left(\mathrm{j} \omega_{F}\right)}$ 对角度不对中的敏 感性来定量判断角度不对中量的严重程度。

\section{参 考 文 献}

[1] 艾延廷，付其，田晶，等. 基于融合信息摘距的转子裂 纹-碰摩耦合故障诊断方法[J]. 航空动力学报，2013， 28(10) : 2161-2166.

AI Yanting , FU Qi , TIAN Jing , et al. Diagnosis method for crack-rubbing coupled fault in rotor system based on integration of information entropy distance[J]. Journal of Aerospace Power , 2013 , 28(10) : 2161-2166.

[2] 时培明，王敬，党会，等. 基于改进 EMD 和 FastICA 的旋转机械耦合故障诊断方法研究 $[\mathrm{J}]$. 现代制造工程, 2016(7) : 12-18

SHI Peiming , WANG Jing , DANG Hui , et al. Study on coupling faults of rotary machinery diagnosis method based improved EMD and FastICA[J]. Modern Manufacturing Engineering , 2016(7) : 12-18.

[3] 陶海亮, 左志涛, 高庆, 等. 基于时频分析的裂纹转子 碰摩故障特征研究[J]. 推进技术, 2013,34(4):520-528. TAO Hailiang, ZUO Zhitao , GAO Qing, et al. Fault analysis of rotor with rub-impact and crack based on time-frequency analysis[J]. Journal of Propulsion Technology , 2013 , 34(4) : 520-528.

[4] 焦卫东, 蒋永华, 施继忠，等. 一种旋转机械系统耦合 故障诊断的新方法 [J]. 仪器仪表学报，2016，37(7) : 1449-1456.

JIAO Weidong ,JIANG Yonghua ,SHI Jizhong ,et al. New approach for diagnosis on coupled faults of rotating machine system[J]. Chinese Journal of Scientific Instrument , 2016 , 37(7) : 1449-1456.

[5] 徐自力, 宷柏通, 范小平, 等. 基于分层模态综合法的 
大型汽轮发电机组转子-末级叶片耦合系统扭转振动分 析[J]. 动力工程学报, 2014, $12: 938-944$.

XU Zili , DOU Baitong, FAN Xiaoping, et al. Coupled torsional vibration analysis of shaft-last stage blade system in large turbo-generator units using CMS method with nested substructures[J]. Power Engineering , 2014 , 12 : 938-944.

[6] XIA X , ZHOU J , LI C , et al. A novel method for fault diagnosis of hydro generator based on NOFRFs[J]. International Journal of Electrical Power \& Energy Systems , $2015,71:$ 60-67.

[7] PETKOVSKA M, DO D D. Use of higher-order frequency response functions for identification of nonlinear adsorption kinetics : Single mechanisms under isothermal conditions[J]. Nonlinear Dynamics , 2000 , 21(4) : 353-376.

[8] 李志农, 杜宜光, 肖尧先. 基于非线性输出频率响应函 数的多裂纹转子故障诊断方法研究 $[\mathrm{J}]$. 兵工学报, 2015 , 36(6) : 1096-1103.

LI Zhinong ,DU Yiguang ,XIAO Yaoxian. Fault diagnosis method of rotor system with multi-crack based on nonlinear output frequency response function[J]. Acta Armamentarii , 2015 , 36(6) : 1096-1103.

[9] ALEVRAS P ,THEODOSSIADES S ,RAHNEJAT H. On the dynamics of a nonlinear energy harvester with multiple resonant zones[J]. Nonlinear Dynamics , 2018 , 92(3) : 1271-1286.

[10] 韩海涛, 马红光, 李飞, 等. 基于系统输出频率响应函 数的非线性系统研究[J]. 工程设计学报, 2011,45(10): 77-81.

HAN Haitao , MA Hongguang , LI Fei , et al. Research on nonlinear system based on output frequency response functions[J]. Journal of Engineering Design , 2011 , 45(10) : 77-81.

[11] 姚红良, 韩清凯, 冯霏, 等. 多自由度局部非线性系统 频域响应及非线性位置的辨识方法 [J]. 动力学与控制 学报, 2011, 9(2): 107-110.

YAO Hongliang ,HAN Qingkai ,FENG Fei ,et al. NOFRF of the locally nonlinear MDOF system and the detection method of the nonlinearity position[J]. Journal of Dynamics and Control , 2011 , 9(2) : 107-110.

[12] 韩清凯, 杨英, 郎自强, 等. 基于非线性输出频率响应 函数的转子系统碰摩故障的定位方法研究 [J]. 科技导 报，2009，27(2):29-32.

HAN Qingkai, YANG Ying, LANG Zhiqiang, et al. A novel method for locating rub-impacts in rotor systems using a nonlinear output frequency response functions[J]. Science \& Technology Review , 2009 , 27(2) : 29-32.

[13] 程长明. 基于 Volterra 级数的非线性系统辨识及其应用
研究[D]. 上海 : 上海交通大学, 2015.

CHENG Changming. Volterra series based nonlinear system identification and it's application[D]. Shanghai :

Shanghai Jiao Tong University , 2015.

[14] RUGH W J. Nonlinear system theory[M]. Charles Village : The Johns Hopkins University Press , 2002.

[15] PENG Z K , LANG Z Q , BILLINGS S A , et al. Analysis of locally nonlinear MDOF systems using nonlinear output frequency response functions[J]. Journal of Vibration and Acoustics , 2009 , 131(5) : 51-63.

[16] 武红霞, 韩捷, 陈宏, 等. 基于 NOFRF 裂纹转子非线 性频谱分析 [J]. 机械强度，2017，39(6)：1271-1274.

WU Hongxia , HAN Jie , CHEN Hong, et al. Nonlinear frequency spectral analysis of cracked rotor based on NOFRF[J]. Journal of Mechanical Strength ,2017,39(6) : 1271-1274.

[17] 杨凯, 张业伟，丁虎，等. 基于非线性输出频响函数的 NES 动力学参数设计 $[\mathrm{J}]$. 振动与冲击 , 2016, 35(21) : $76-80,86$.

YANG Kai ,ZHANG Yewei ,DING Hu , et al. Parametric design of nonlinear energy sinks based on nonlinear output frequency-response functions $[\mathrm{J}]$. Journal of Vibration and Shock , 2016 , 35(21) : 76-80, 86.

[18] PENG Z K , LANG Z Q , BILLINGS S A , et al. Analysis of bilinear oscillators under harmonic loading using nonlinear output frequency response functions[J]. International Journal of Mechanical Sciences , 2007 , 49(11) : 1213-1225.

[19] 陈果, 李成刚, 王德友. 航空发动机转子-滚动轴承-支 承-机匣耦合系统的碰摩故障分析与验证 [J]. 航空动力 学报, 2008, 23(7): 1304-1311.

CHEN Guo, LI Chenggang, WANG Deyou. Nonlinear dynamic analysis and experiment verification of rubbing fault s of rotor-ball bearing-support-stator coupling system for aero-engine[J]. Journal of Aerospace Power , 2008 , 23(7) : 1304-1311.

[20] XU M , MAZANGONI R D. Vibration analysis of a motorflexible coupling rotor system subject to misalignment and unbalance : Part I - Theoretical model and analysis[J]. Journal of Sound and Vibration , 1994 , $176: 663-679$.

[21] XU M , MAZANGONI R D. Vibration analysis of a motorflexible coupling rotor system subject to misalignment and unbalance : Part2 - Experimental validation [J]. Journal of Sound and Vibration ,1994, 176 : 681-691.

作者简介: 李志农, 男, 1966 年出生, 博士, 教授。主要研究方向为转 子动力学, 机械故障诊断。

E-mail : lizhinong@tsinghua.org.cn 